\title{
Dynamics of the inner edge of the dead zone in protoplanetary disks
}

\author{
Julien Faure ${ }^{1,2}$, Sebastien Fromang ${ }^{1,2}$ and Henrik Latter ${ }^{3}$ \\ ${ }^{1}$ CEA, Irfu, SAp, Centre de Saclay, F-91191 Gif-sur-Yvette, France; ${ }^{2}$ UMR AIM, \\ CEA-CNRS-Univ. Paris VII, Centre de Saclay, F-91191 Gif-sur-Yvette, France; ${ }^{3}$ Department \\ of Applied Mathematics and Theoretical Physics, University of Cambridge, Centre for \\ Mathematical Sciences, Wilberforce Road, Cambridge, CB3 0WA, UK
}

\begin{abstract}
In protoplanetary disks, the inner boundary between an MRI active and inactive region has recently been suggested to be a promising site for planet formation. A set of numerical simulations has indeed shown that vortex formation mediated by the Rossby wave instability is a natural consequence of the disk dynamics at that location. However, such models have so far considered only the case of an isothermal equation of state, while the complex thermodynamics is at the heart of how this region works. Using the Godunov code Ramses, we have performed 3D global numerical simulations of protoplanetary disks that relax the isothermal hypothesis. We find that, at the interface, the disk thermodynamics and the turbulent dynamics are intimately entwined, because of the importance of turbulent dissipation and thermal ionisation.
\end{abstract}

\section{Introduction}

We aim to investigate the interplay of thermodynamics and dynamics in the inner regions of protoplanetary disks. We then simulate the feedback of temperature on turbulence via the thermal heating with a set of numerical experiments of MRI turbulence. To complete the thermodynamical feedback loop, we link the resistivity to the temperature using, for simplicity, a step function. Such a form capture the strong sensitivity of resistivity in Saha's law around the threshold $T_{M R I}$.

\section{Results}

Initially, the gas is in a turbulent state from $R=1.5$ to $R=3.5$ and a dead zone extends from $R=3.5$ to the outer boundary of the simulation domain.

We first consider the case of a resistivity that is a function of position only. 900 orbits after the beginning of the simulation, the temperature has reached a quasi steady state. We check that the flow is laminar in the dead zone region. However, we note that, at this location, the temperature (Fig. 1) does not drop to the minimum value, as one might assume in absence of turbulent heating. The curve on the (Fig. 1) establishes that the increased temperature is mostly due to density waves shocking inside the dead zone.

We then move to the more realistic case in which the resistivity is self-consistently calculated as a function of temperature. We restart the simulation after closing the thermodynamical feedback loop. The left panel of Fig. 2 shows the radial profile of the turbulent activity throughout the simulation. We see that the interface between the active and the dead zone is not static: a MRI front propagates outward over several disk scale heights. This dynamical behavior had been anticipated by previous exploratory works which modelled the complicated interaction between turbulence and temperature via a crude diffusive process (Latter \& Balbus, 2012). On the right panel of Fig. 2, we show the 


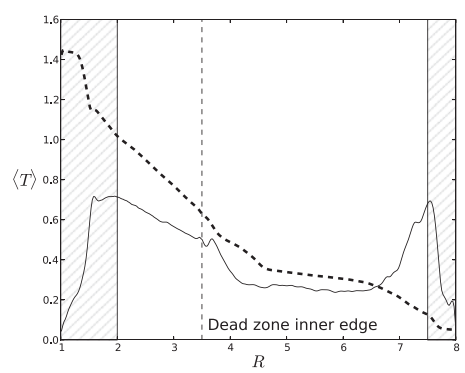

Figure 1. Temperature radial profile in the MHD simulation (black dashed line). The equilibrium temperature profile balancing the wave heating is shown by the plain black line.
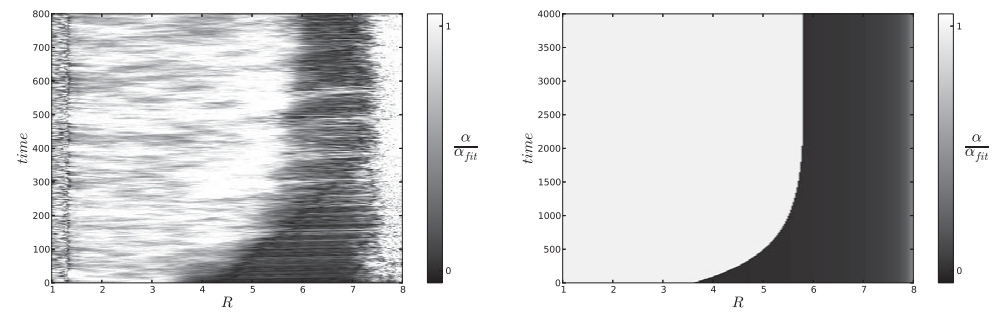

Figure 2. Turbulent activity evolution, in the MHD simulation (left panel), in the mean field simulation (right panel)

result obtained using the mean field model of Latter \& Balbus (2012) initialized similarly to our MHD simulation. The two fronts propagate outward and stop at the same critical radius. The two y-axis scales indicate the front seen in the MHD simulation propagates much faster than the other one.

\section{Conclusions}

We have shown that the active zone controls the dead zone thermodynamics via waves excited at the interface. These waves transport energy, heating up the gas deeply inside the dead zone. The good agrement between the two final front position demonstates the mean field model can be used to understand the front propagation engine: A front propagates outward (inward) if the thermal energy reservoir before the interface is (not) sufficient to support dead zone thermal feeding until temperature exceed the ionization threshold. Hence, the final front position can be predicted once knowing the disk thermal structure only. By contrast, the velocity of the front is sensitive to the thermal flux connecting the active zone to the dead zone. A diffusive description of that flux fails at explaining the front velocities as it induces much too slow fronts. Instead, fronts move rapidly because waves efficiently transport energy across the interface. This emphasizes their importance for the dead zone dynamics as well.

For near future, we plan to investigate how vortices, known to form at the interface, are sensitive to the thermodynamical interplay we described here.

\section{References}

Latter, H. N., \& Balbus, S. 2012, MNRAS, 424, 1977-1990 